\title{
Piedra Branca por Trichosporon ovoides no Sudeste do Brasil: Relato e Breve Revisão da Literatura
}

\author{
Flávia Regina Ferreira', Talita Pollo², Marina Thereza Fogo Pereira ${ }^{3}$, Margareth Neila Prats Bueri ${ }^{4}$ \\ 'Mestre e Doutora em Ciências pela Universidade Federal de São Paulo - UNIFESP/EPM, Professora Assistente III da disciplina \\ de Dermatologia do Departamento de Medicina da Universidade de Taubaté / Médica do Serviço de Dermatologia do Hospital \\ Universitário de Taubaté - HUT/UNITAU, Taubaté-SP, Brasil \\ ${ }^{2}$ Médica, Especializanda do $3^{\circ}$ ano de Dermatologia do Hospital Universitário de Taubaté, HUT/UNITAU, Taubaté-SP, Brasil \\ ${ }^{3}$ Médica, Especializanda do $7^{\circ}$ ano de Dermatologia do Hospital Universitário de Taubaté - HUT/UNITAU, Taubaté-SP, Brasil \\ ${ }^{4}$ Bióloga, Laboratório MIL, Taubaté-SP, Brasil
}

RESUMO - A raridade desta entidade e a exuberância destes casos de piedra branca, associada à documentação completa (incluindo a identificação da espécie) motivaram este relato. Mãe e filha apresentando nódulos diminutos, em torno de 1 a 2 mmde diâmetro, coloração branco-amarelada na haste dos cabelos, há cerca de 6 meses. Assintomáticas. $\bigcirc$ clima quente e húmido e o hábito do uso de cremes recondicionadores podem ter sido fatores determinantes da infecção. A análise dos fios ao microscópio óptico, a cultura e o microcultivo e a espectrometria de massas (MALDI-TOF/MS) concluíram o diagnóstico de piedra branca por Trichosporon ovoides. O tratamento de escolha foi o corte dos cabelos associado a champô de cetoconazol $2 \%$. PALAVRAS-CHAVE - Brasil; Cetoconazol/uso terapêutico; Dermatoses do Couro Cabeludo; Piedra/diagnóstico; Trichosporon.

\section{White Piedra by Trichosporon ovoides in Southeastern Brazil: Report and Brief Literature Review}

ABSTRACT - The rarity of this entity and the exuberance of these cases of white piedra, associated with the complete documentation (including species identification) motivated this report. Mother and daughter presenting asymptomatic tiny white-yellowish nodules, around 1 to $2 \mathrm{~mm}$ in diameter, on the hair shaft, for about 6 months. The hot and humid weather and the habit of using conditioning creams may have been determining factors of the infection. Analysis of the hair strands under an optical microscope, culture and microculture and mass spectrometry (MALDI-TOF/MS) concluded the diagnosis of white piedra by Trichosporon ovoides. It resolved with a short haircut associated with ketoconazole $2 \%$ shampoo.

KEYWORDS - Brazil; Piedra/diagnosis; Ketoconazole/therapeutic use; Scalp Dermatoses; Trichosporon.

\section{INTRODUÇÃO}

A piedra branca (PB) é uma infecção fúngica crónica, rara e assintomática da cutícula do pelo, descrita originalmente por Beigel em 1865..$^{1-6} \mathrm{O}$ agente etiológico foi inicialmente descrito como Sclerotium beigellii e depois substituído por Trichosporon beigelii.' O género Trichosporon encontra-se sob constante revisão, sendo atualmente formado por 51 espécies, das quais 16 estão envolvidas em quadros clínicos. São 6 as espécies de maior relevância: T. asahii, T. asteroides, T. cutaneum (sinónimo de T. beigelii), T. mucoides, T. inkin e $T$. ovoides. ${ }^{2,3,5}$ As duas últimas espécies são comumente envolvidas em casos de PB, variante genital e do couro cabeludo, respectivamente, e as demais espécies são responsáveis por otomicoses, ceratite micótica, pneumonites, endocardite, encefalite, hepatite, peritonites, envolvimento renal e infecções disseminadas, sendo que nesses casos estas infecções são denominadas de tricosporonoses. . $^{2,3,6}$

A PB é encontrada em regiões de clima temperado e tropical afetando ambos os sexos e todos os grupos etários. ${ }^{2,7}$ modo de transmissão ainda não é bem conhecido, porém dentre os fatores predisponentes estão o calor, humidade, imunossupressão, antibióticos e procedimentos invasivos.
Correspondência: Flávia Regina Ferreira

Rua Paraguai, 59 - Jardim das Nações

Taubaté-SP, Brasil - CEP: 12030-240

E-mail:dermagica@uol.com.br

DOI: https://dx.doi.org/10.29021/spdv.77.2.1038
Recebido/Received

17 Janeiro/January 2019

Aceite/Accepted

17 Abril/April 2019 


\section{Caso Clínico}

Alguns autores consideram como fonte provável de transmissão animais domésticos e a via sexual. ${ }^{4}$

Clinicamente caracteriza-se pela presença de nódulos firmes e irregulares de coloração esbranquiçada a acastanhada, assintomáticos, comprometendo a haste de pelos das áreas genitais, das axilas, barba e bigode, menos frequentemente do couro cabeludo, cílios e sobrancelhas. Não há alteração no folículo piloso, mas podem encontrar-se lesões eritemato-descamativas na pele subjacente. ${ }^{2}$

Entre os diagnósticos diferenciais encontram-se a piedra preta, que apresenta nódulo endurecido e aderente ao fio do cabelo, a pediculose, a tricomicose axilar e anormalidades da haste do cabelo, como a tricorrexis nodosa, moniletrix e tricoptilose. ${ }^{3,8}$ Quando há comprometimento da área subjacente podemos acrescentar aos diagnósticos diferenciais as dermatofitoses, as candidíases e o eritrasma. ${ }^{2}$

O diagnóstico laboratorial é realizado através da análise dos pelos afetados ao microscópio óptico, utilizando-se hidróxido de potássio de $10 \%$ a $40 \%$ em solução aquosa, em que se observam elementos micelianos (artroconídios e blastoconídios, binômio que caracteriza o género Trichosporon) em forma de nódulos dispostos perpendicularmente à superfície dos pelos. ${ }^{2,4}$ A cultura em meio de ágar Sabouraud sem actidiona à temperatura ambiente, demonstra o crescimento moderado de colônia branco-amarelada, pregueada e com aparência de cera, posteriormente adquirindo coloração acinzentada. O microcultivo da colônia mostra hifas hialinas, artroconídios e blastoconídios. ${ }^{2,9} \bigcirc$ género Trichosporon não fermenta carbohidratos, mas assimila glicose, galactose, sacarose, maltose, trealose (provavelmente) e lactose, além de ser urease-positivo. 9 Para a identificação das espécies são utilizados critérios morfológicos, provas bioquímicas, de aglutinação de antígeno, técnicas analíticas (espectrometria de massas) e técnicas moleculares. $^{3}$

O tratamento de escolha da PB é cortar os pelos afetados e, devido à recorrência frequente, são indicados antifúngicos tópicos, como imidazóis, ciclopiroxolamina e piritionato de zinco. ${ }^{1-5,7,9}$

\section{CASO CLÍNICO}

Mulher de 29 anos, fototipo III, residente na cidade de Taubaté-SP com queixa de pontos brancos nos cabelos há aproximadamente 6 meses. Acompanhava-a a filha de 7 anos com quadro semelhante. Ao exame dermatológico de ambas, observavam-se nódulos diminutos, em torno de 1 a $2 \mathrm{~mm}$ de diâmetro, coloração branco-amarelada, aderentes à haste dos fios de cabelo do couro cabeludo, assintomáticos e sem alterações no folículo piloso e pele subjacente. Para melhor visualização das lesões foi realizada dermatoscopia dos cabelos (Fig. 1).

A análise dos fios afetados à microscopia óptica da criança e da mãe permitiu a visualização de nódulos branco-amarelados, macios, aderentes à haste capilar e dispostos ao redor desta sem a alterar, de modo perpendicular, corroborando a hipótese clínica de PB (Fig. 2).

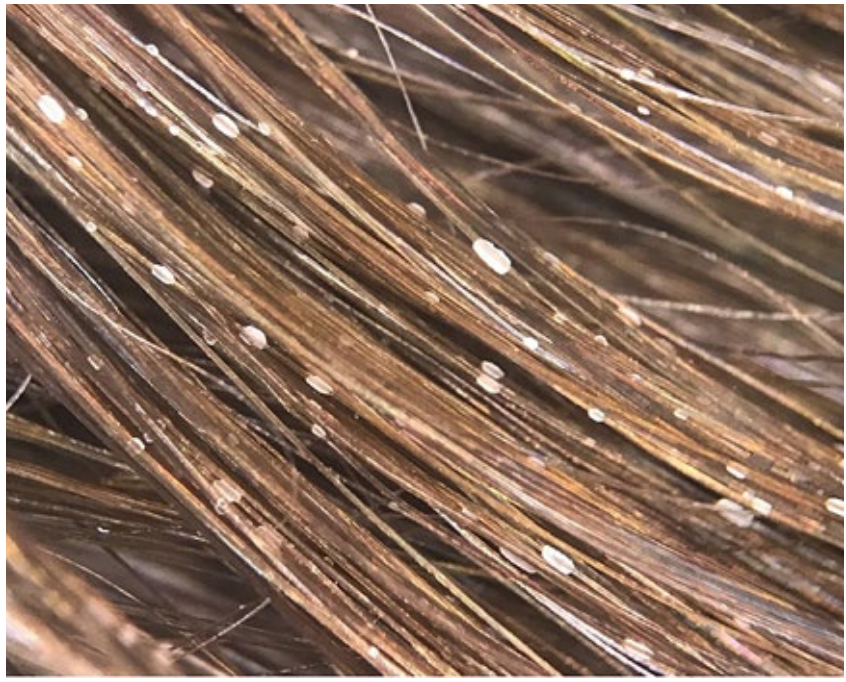

Figura 1 - Dermatoscopia dos fios de cabelos

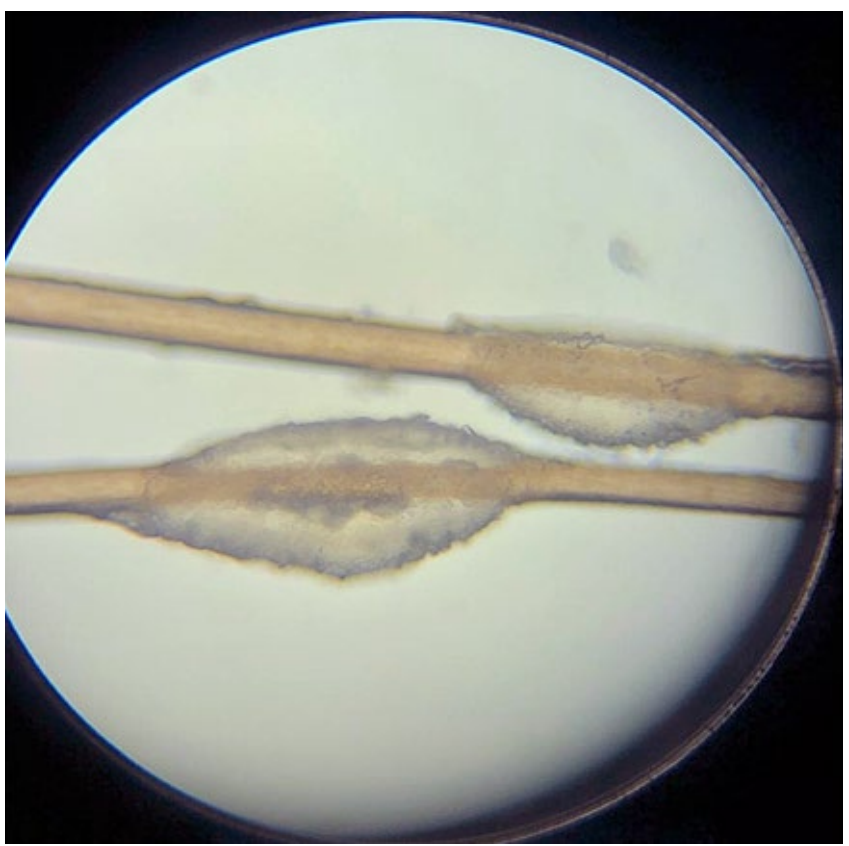

Figura 2 - Microscopia óptica: nódulos branco-amarelados, macios, aderidos à haste capilar sem a alterar, de modo perpendicular.

A cultura dos pelos em meio ágar Sabouraud demostrou macroscopicamente o crescimento de colónia leveduriforme, de cor branco-amarelada, pregueada, com aparência de cera (Fig. 3) e o microcultivo evidenciou hifas septadas, blastoconídios e artroconídios característicos do género Trichosporon spp. (Fig. 4). A identificação taxonómica da espécie foi realizada através de espectrometria de massa (MALDI-TOF/ MS) identificando o Trichosporon ovoides na haste dos cabelos de mãe e filha.

O tratamento proposto foi o corte dos cabelos e a utilização de champô de cetoconazol durante 30 dias. 


\section{Caso Clínico}

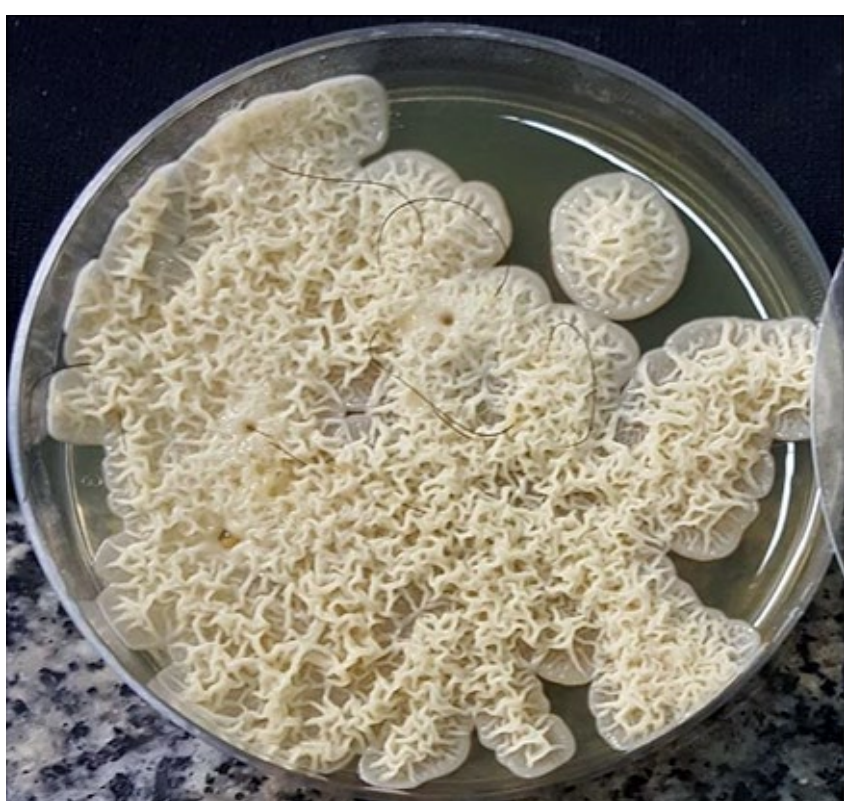

Figura 3 - Cultura em ágar Sabouraud: colônia leveduriforme, de cor branco-amarelada, pregueada, com aparência de cera.

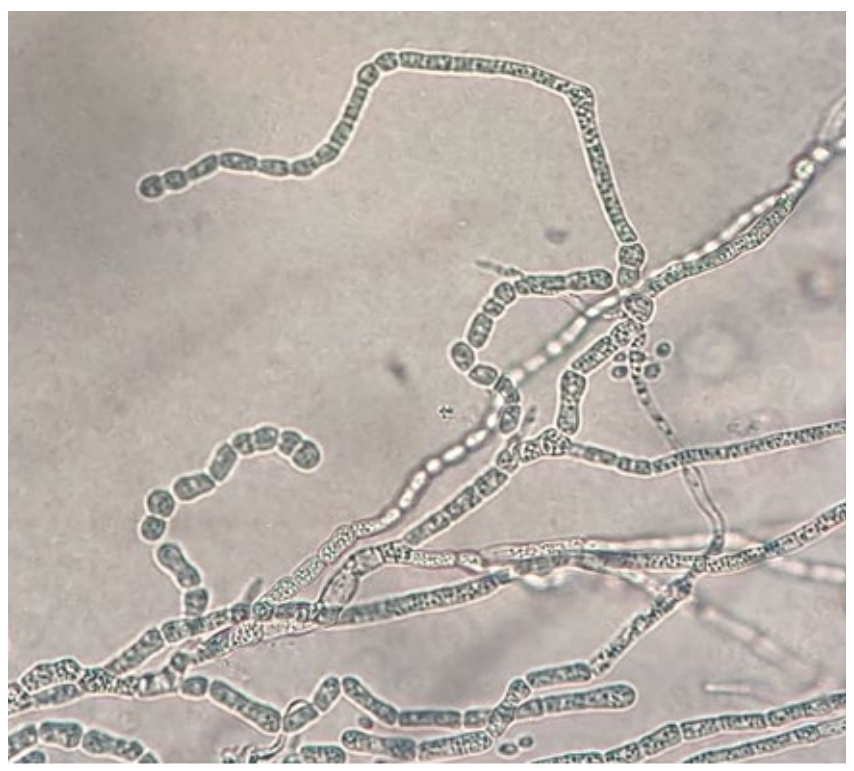

Figura 4 - Microcultivo: hifas septadas, blastoconídios e artroconídios característicos do genero Trichosporon spp.

\section{DISCUSSÃO}

A PB é uma infecção fúngica rara, ocasionada por espécies de leveduras do género Trichosporon, tendo sido relatada na América do Sul, América do Norte, Sul da Ásia, Médio Oriente, Europa, Japão e Austrália. No Brasil há referência de casos na Paraíba, Rio de Janeiro, São Paulo, Belém e Espírito Santo. A maioria dos casos brasileiros de PB localizam-se nas áreas genitais e lesões no couro cabeludo foram relatadas no Rio de Janeiro, Paraíba e São Paulo. 2,3
O género Trichosporon foi considerado inicialmente como representado por uma única espécie ( $T$. beigelli), sendo atribuída a esta várias denominações. ${ }^{3}$ Baseado em análises moleculares do rDNA, Guého et al (1992) propuseram que o género Trichosporon fosse formado por espécies distintas. ${ }^{10}$

$\bigcirc T$. cutaneum (T. beigelli) é uma levedura saprofítica e tem como habitat natural solos, águas e vegetais, mas já foi encontrado também em macacos e cavalos. ${ }^{1,4,11}$ No ser humano pode fazer parte do microbioma normal da pele, unha, mucosa oral e na região genital, onde pode ser causa de PB genital em homens jovens e também ser observado em sinergismo com a bactéria Coryneform, participante da flora normal dessa região. $3,7,8$

T. ovoides causa a PB do couro cabeludo, o qual raramente é acometido, enquanto o T. inkin é amplamente detectado na piedra púbica. O T. asteroides é isolado com menor frequência em lesões superficiais e, na maioria das vezes, é provavelmente contaminante. ${ }^{11} \mathrm{O}$ T. asahii e o T. mucoides são fungos patogénicos sistémicos, encontrando-se especialmente associados a situações de imunossupressão. A tricosporonose sistémica pode levar a febre, pneumonites, endocardite, celulite, glomerulonefrite, endoftalmite, abscesso cerebral, otomicose, onicomicose e infecções disseminadas. $3,4,11$

Dessa forma, a identificação das espécies torna-se um aspecto importante. A correta caracterização dessas leveduras também é importante para seleção do tratamento antifúngico, tendo em vista que isolados de T. asahii podem apresentar uma resistência maior a anfotericina B quando comparado aos triazóis. Todavia, as demais espécies do género são mais resistentes aos triazóis. ${ }^{3,12}$

Ressaltamos a escassez de literatura sobre a identificação de espécies. A identificação de leveduras por métodos clássicos é demorada e por vezes inconclusiva. Técnicas de biologia molecular têm sido empregadas e, métodos analíticos, como a espectrometria de massas (MALDI-TOF/ MS), têm-se mostrado promissores para a identificação e classificação de microrganismos (entre eles leveduras artroconidiadas, incluindo espécies de Trichosporon) por sua rapidez e precisão. ${ }^{13-15}$

De ocorrência preferencialmente tropical, a infecção e a manutenção desta tricopatia possivelmente está relacionada ao hábito do uso de produtos oleosos que, aliados às condições ambientais, como clima quente e húmido, favoreçam o desenvolvimento da doença, fatores estes referidos e observados nas pacientes deste estudo. Também foi sugerido que fatores individuais de cada indivíduo (cabelos étnicos, comprimento médio ou longo dos fios, entre outros), o contato com materiais contaminados / água estagnada e animais infectados podem transmitir esta condição clínica. Ainda, fatores relacionados com a sudorese excessiva, hábitos como prender os cabelos molhados ou húmidos, o uso de cremes e condições de base do indivíduo (imunodeficiências) estão intimamente ligados ao desenvolvimento desta tricopatia. $^{2-4,11} \bigcirc$ padrão socioeconômico e a associação com falta de higiene pessoal na PB são controversos na literatura. ${ }^{2}$ 
Apesar da ocorrência em ambos os sexos, existe um aparente predomínio no sexo feminino, conforme os achados de Pontes et al e Diniz e Souza Filho, concordando com o observado neste relato. ${ }^{2,8}$

O tratamento recomendado é cortar os cabelos e utilizar champôs à base de piritionato de zinco a $2 \%$ ou cetoconazol a $2 \%$ durante 30 dias, sendo observado no final desse tempo regressão das lesões. ${ }^{1-5,7,9}$ Agentes sistémicos relatados incluem cetoconazol, itraconazol, fluconazol, voriconazol, posaconazol e anfotericina $B$, mas são mais utilizados nas tricosporonoses sistêmicas. ${ }^{4,5,12}$

Conflitos de interesse: Os autores declaram não possuir conflitos de interesse.

Suporte financeiro: $O$ presente trabalho não foi suportado por nenhum subsídio ou bolsa.

Confidencialidade dos dados: Os autores declaram ter seguido os protocolos do seu centro de trabalho acerca da publicação dos dados de doentes.

Direito a privacidade e consentimento escrito: Os autores declaram que pediram consentimento para usar as imagens no artigo.

Conflicts of interest: The authors have no conflicts of interest to declare.

Financing Support: This work has not received any contribution, grant or scholarship.

Confidentiality of data: The authors declare that they have followed the protocols of their work center on the publication of data from patients.

Privacy policy and informed consent: The authors declare that have the written informed consent for the use of patient's photos in this article.

Proveniência e revisão por pares: Não comissionado; revisão externa por pares

Provenance and peer review: Not commissioned; externally peer reviewed

\section{REFERÊNCIAS}

1. Zaitz C, Campbell I, Marques AS, Luiz LRB, Souza VM. Compêndio de micologia médica. Rio de Janeiro: MEDSI; 1998.

2. Diniz LM, Souza Filho JB. Estudo de 15 casos de piedra branca observados na Grande Vitória (Espírito Santo - Brasil) durante cinco anos. An Bras Dermatol. 2005; 80:49-52.

3. Inácio CP. Modelo de Infecção in vitro da piedra branca, análise dos aspectos morfológicos, ultraestruturais e abordagem de identificação polifásica dos agentes etiológicos. [Dissertação de Mestrado em Biologia de Fungos]. Recife: Universidade Federal de Pernambuco; 2015.

4. Araújo IR, Marques SG. Tricosporonose: Etiologia, manifestações clínicas, diagnósticos laboratoriais, epidemiologia e tratamento [acesso em 14 jan 2019]. Disponível em: http://www.congressos.ifal.edu.br/ index.php/connepi/CONNEPI2010/paper/viewFile/1134/634.

5. Colombo AL, Padovan ACB, Chaves GM. Current knowledge of Trichosporon spp. and Trichosporonosis. Clin Microbiol Rev. 2011; 24:682-700. doi: 10.1128/ CMR.00003-11.

6. Rodríguez YP, Govea YS, Martori MF, Lorenzo AG, Rodríguez JLA. Piedra Blanca en Cuero Cabelludo. Primer Reporte en Matanzas, Cuba. Folia Dermatológica Cubana [periódicos na Internet]. 2014 [acesso em 14 jan 2019]; 8(2). Disponível em: http://bvs.sld.cu/revistas/fdc/vol8_2_14/fdc06214.htm

7. Drake LA, Dinehart SM, Farmer ER, Goltz RW, Graham GF, Hordinsky MK, et al. Guidelines of care for superficial mycotic infections of the skin: Piedra. J Am Acad Dermatol. 1996; 34:122-4.

8. Pontes ZB, Ramos AL, Lima EO, Guerra MF, Oliveira NM, Santos JP. Clinical and Mycological Study of Scalp White Piedra in the State of Paraíba, Brazil. Mem Ins Oswaldo Cruz. 2002; 97: 747-50.

9. Lacaz CS, Porto E, Heins Vaccari EM, Melo NT. Guia para identificação: Fungos, Actinomicetos, Algas de interesse médico. São Paulo: Sarvier; 1998.

10. Gueho E, Smith MT, De Hoog GS, Billongrande G, Christen R, Batenburg-Van Der Vegte WH. Contributions to a revision of the genus Trichosporon. Antonie Van Leeuwenhoek. 1992; 61:289-316.

11. Tambe SA, Dhurat SR, Kumar CA, Thakare P, Lade N, Jerajani $\mathrm{H}$, et al. Two cases of scalp white piedra caused by Trichosporin ovoides. Indian J Dermatol Venereol Leprol. 2009; 75:293-95.

12. Taverna CG, Córdoba S, Murisengo OA, Vivot W, Davel G, Bosco-Borgeat ME. Molecular identification, genotyping, and antifungal susceptibility testing of clinically relevant Trichosporon species from Argentina. Med Mycol. 2014; 52:356-66.

13. Assis DM, Juliano L, Juliano MA. Espectometria de massas aplicada na classificação e identificação de microorganismos. Rev Univ Vale do Rio Verde. 2011; 9:344-55.

14. Croxatto A, Prod'hom G, Greub G. Applications of MALDI-TOF mass spectrometry in clinical diagnostic microbiology. FEMS Microbiol Rev. 2012; 36:380-407. doi: 10.1111/j.1574-6976.2011.00298.x.

15. Kolecka A, Khayhan K, Groenewald M, Theelen B, Arabatzis $M$, Velegraki $A$, et al.Identification of medically relevant species of arthroconidial yeasts by use of matrix-assisted laser desorption ionization-time of flight mass spectrometry. J Clin Microbiol. 2013; 51:2491500. doi: 10.1128/JCM.00470-13. 